\title{
Radiação gama e tempo de armazenamento sobre a oxidação lipídica, cor objetiva, pigmentos heme e nitrito residual de mortadelas elaboradas com diferentes níveis de nitrito
}

\author{
Gamma radiation and storage time on lipidic oxidation, color, heme pigments and residual nitrite \\ of bologna-type sausages formulated with different nitrite additions
}

\author{
Monalisa Pereira Dutra ${ }^{\mathrm{I}}$ Eduardo Mendes Ramos ${ }^{\mathrm{I}}{ }^{*}$ Alcinéia de Lemos Souza Ramos ${ }^{\mathrm{II}}$ \\ Paulo Rogério Fontes ${ }^{\mathrm{II}}$ Giselle Pereira Cardoso ${ }^{\mathrm{I}}$ Alexandre Soares Leal ${ }^{\mathrm{II}}$
}

\section{RESUMO}

Este trabalho teve como objetivo avaliar os efeitos da aplicação de diferentes doses de irradiação (0, 7,5 e 15kGy) e níveis de nitrito $(0,75$ e 150ppm) sobre o índice de TBARS, cor objetiva, concentração residual de nitrito e de pigmentos heme totais (PHT) e nitrosos (PHN) em mortadelas armazenadas sob refrigeração $\left(4^{\circ} \mathrm{C}\right)$ por 69 dias. A irradiação aumentou $(P<0,05)$ os valores de TBARS e reduziu $(P<0,05)$ os níveis de nitrito residual no primeiro mês de estocagem, mas pouco afetou os níveis de PHN das amostras. A cor dos produtos formulados com 150ppm de nitrito não foi afetada $(P>0,05)$ pela irradiação. Pôde-se concluir que altas doses de irradiação podem ser aplicadas em mortadelas com mais de $75 \mathrm{ppm}$ de nitrito, sem que haja grandes alterações na cor e nas características químicas dos produtos.

Palavras-chave: produto emulsionado, índice de TBARS, pigmentos nitrosos.

\section{ABSTRACT}

The present study was undertaken to evaluate the irradiation doses $(0,7,5$ and $15 k G y)$ and nitrite levels $(0,7,5$ and $15 k G y)$ effects on the TBARS index, objective color $(C I E L A B)$, residual nitrite content and total and nitrous heme pigments in bologna-type sausage during storage $\left(4^{\circ} \mathrm{C}\right)$ for 69 days. Irradiation increased $(P<0.05)$ TBARs values and decreased $(P<0.05)$ residual nitrite content in the first month of storage, but had little effects on nitrous heme pigments content. The color of products with 150ppm nitrite added was not affected $(P>0.05)$ by irradiation. Results indicated that high doses of gamma radiation can be applied in bologna-type sausages with 75 ppm nitrite added without major changes in color and chemical characteristics.

Key words: emulsion-type cooked product, TBARS values, nitrous heme pigment.

\section{INTRODUÇÃO}

Sais de nitrito e nitrato são adicionados em produtos cárneos para conferir a cor rósea e o sabor característicos de produtos curados, além de prevenir alterações desagradáveis oriundos da rancidez oxidativa dos lipídios e atuar como conservante, principalmente contra o crescimento e a produção de toxina do Clostridium botulinum (CASSENS, 1997). Entretanto, o uso de nitrito em produtos cárneos está relacionado com a formação de compostos $\mathrm{N}$-nitrosos potencialmente tóxicos, mutagênicos e carcinogênicos, como as N-nitrosaminas (HOUSER et al., 2003). Dessa forma, pesquisas têm buscado formas alternativas para substituir ou reduzir as quantidades adicionadas de nitrito nos processos de transformação da carne em produtos.

No Brasil, a quantidade residual máxima de nitrito, expressa como nitrito de sódio, permitida para

'Programa de Pós-graduação em Ciência dos Alimentos, Departamento de Ciência dos Alimentos (DCA), Universidade Federal de Lavras (UFLA), Lavras, MG, Brasil.

"DCA, UFLA, 37200-000, Lavras, MG, Brasil. E-mail: emramos@dca.ufla.br. *Autor para correspondência.

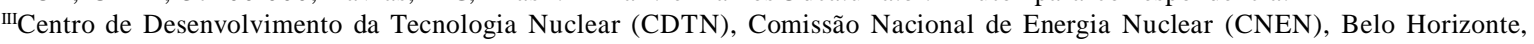
MG, Brasil. 
produtos curados é de $150 \mathrm{ppm}$. Sua redução, no entanto, é limitada pelos riscos relativos ao desenvolvimento do $\boldsymbol{C}$. botulinum. Uma alternativa viável é o uso da radiação gama, reconhecida como a melhor tecnologia para a destruição microbiana em alimentos (BREWER, 2004).

Estudos têm relatado que a radiação gama induz a radiólise do nitrito e das N-nitrosaminas, reduzindo a concentração desses componentes no produto final (AHN et al., 2004; JO et al., 2003). No entanto, a irradiação de alimentos também pode promover a formação de radicais livres, resultando na possibilidade de mudanças na cor, oxidação de lipídeos e geração de odor estranho, o que implica respostas negativas para o consumidor quanto à qualidade do produto (BREWER, 2004; BREWER, 2009).

Muitos estudos abordam os impactos da aplicação de baixas doses $(<5 \mathrm{kGy})$ de irradiação em produtos cárneos, embora, na maioria dos países, inclusive no Brasil (BRASIL, 2001), a radiação gama possa ser aplicada em qualquer dose, desde que não comprometa as propriedades funcionais e, ou, sensoriais. Assim, objetivou-se avaliar os efeitos da adição de nitrito e da irradiação gama nos atributos relacionados com a cor de mortadelas durante o armazenamento refrigerado.

\section{MATERIAL E MÉTODOS}

A elaboração das mortadelas seguiu a seguinte formulação: carnes de acém bovino $(42,2 \%)$ e de paleta suína (15,8\%); toucinho (14,0\%); água/gelo (20,0\%); sal (1,9\%); fécula de mandioca (5,0\%); ácido ascórbico $(0,05 \%)$; polifosfato Fosmax $320(0,5 \%$; New Max Industrial ${ }^{\oplus}$ ) e condimento para mortadela 913 $\left(0,5 \%\right.$; New Max Industrial $\left.{ }^{\circledR}\right)$. As matérias-primas cárneas foram obtidas no comércio local, em embalagens lacradas com selo de Inspeção Federal. As mortadelas foram elaboradas com a adição de diferentes níveis de nitrito de sódio (0,75 e 150ppm), sendo processadas em cutter (Mainca, MD-40 BL), embutidas (gomos de $\pm 400 \mathrm{~g}$ ) em tripa artificial sintética (67mm diâmetro, Startripz 67 vermelha, marca Spel) e cozidas por imersão em água até $73^{\circ} \mathrm{C}$ (no ponto frio). Após cozimento, foram resfriadas e estocadas em câmara fria $\left(4^{\circ} \mathrm{C}\right)$ por 24 horas.

Os produtos acabados foram acondicionados em caixas térmicas e submetidas a diferentes doses de irradiação (7,5 e 15kGy), em Irradiador Gama GB-127 (IR-214; MDS Nordion; com fonte de cobalto-60 e taxa de 2,5 $\mathrm{kGy} \mathrm{h}^{-1}$ ), no Centro de Desenvolvimento da Tecnologia Nuclear (Belo Horizonte, MG). As amostras controle (não-irradiadas) foram mantidas nas mesmas condições de temperatura e por períodos de tempo similares às irradiadas. Depois de irradiados, os produtos foram armazenados a $4^{\circ} \mathrm{Ce}$ periodicamente $(0,14,27,41,55$ e 69 dias $)$ coletados para condução das análises.

Cada mortadela foi fatiada ao meio e a superfície interna avaliada por um colorímetro Chroma Meters CR-300 (Konica Minolta Sensing Inc.), sendo os índices de cor $\left(L^{*}, a^{*} \mathrm{e} b^{*}\right)$ obtidos utilizando oiluminante D65 e considerando o valor médio de cinco leituras realizadas em diferentes pontos da superfície (RAMOS \& GOMIDE, 2007). Após avaliação da cor, realizaram-se, em triplicata, as análises de nitrito residual (AOAC, 1995) e pigmentos heme totais (PHT) e nitrosos ( $\mathrm{PHN}$ ), pelo método de Hornsey (RAMOS \& GOMIDE, 2007).

Os tratamentos foram dispostos em um delineamento inteiramente casualisado (DIC), em esquema fatorial $3 \times 3 \times 6$, sendo três concentrações de nitrito $(0,75$ e $150 \mathrm{ppm})$, três níveis de irradiação $(0,7,5$ e $15 \mathrm{kGy})$ e seis tempos de estocagem refrigerada $(0,14$, $27,41,55$ e 69 dias). O experimento foi realizado em duas repetições e os dados submetidos à ANOVA. Foi definido um nível de significância de 5\% e a comparação entre médias, quando pertinente, realizada pelo teste de Tukey.

\section{RESULTADOS E DISCUSSÃO}

Não houve interação significativa $(P>0,05)$ entre doses de irradiação, níveis de nitrito e tempo de armazenamento para o índice de TBARS. No entanto, os valores de TBARS foram maiores $(P<0,05)$ nas amostras que sofreram maiores doses de irradiação (Figura 1), o que era esperado, visto que a irradiação promove a radiólise da água, ocasionando a formação de radicais livres e, consequentemente, favorecendo a oxidação lipídica (BREWER, 2009).

No presente experimento, diferenças nos valores de TBARS não foram observadas $(P>0,05)$ entre as amostras com e sem nitrito. Uma vez que a oxidação lipídica é geralmente dependente da dose de radiação (BYUN et al., 2002), é provável que a quantidade de nitrito adicionada (150 ppm) não tenha sido suficiente para retardar a oxidação ocasionada pela aplicação de maiores doses de radiação (7,5 e 15kGy).

Para a concentração residual de nitrito, houve efeito significativo $(P<0,05)$ apenas para as interações tempo $\mathrm{x}$ níveis de nitrito e tempo $\mathrm{x}$ doses de irradiação (Figura 2). A quantidade de nitrito residual no tempo zero foi maior $(P<0,05)$ nas amostras com maior quantidade de nitrito $(150 \mathrm{ppm})$, apenas se igualando $(P>0,05)$ às amostras adicionadas de 75ppm após 69 dias (Figura 2A), quando atingiu valores médios de 10,66ppm. 


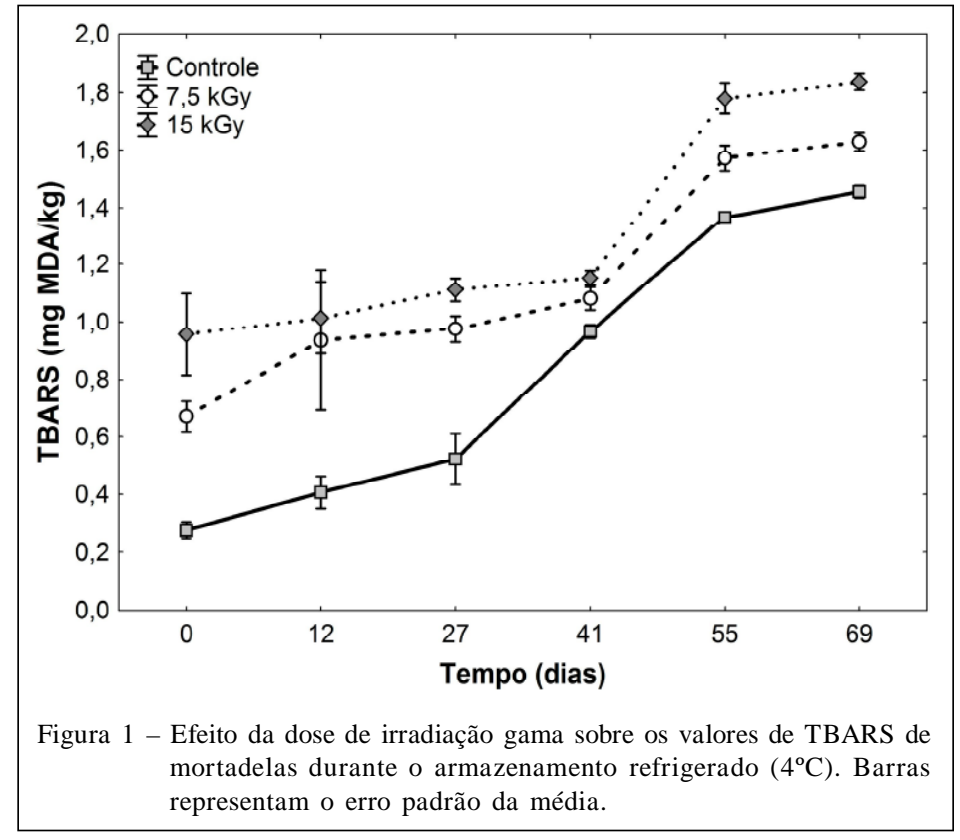

A taxa de queda dos níveis de nitrito residual com o tempo de armazenamento foi similar para as diferentes doses aplicadas (Figura 2B). Segundo SIMIE (1983), a redução do nitrito residual pela irradiação ocorre, provavelmente, pela sua reação com radicais hidroxil produzidos pela radiólise da água.

Embora não tenha sido observada diferença significativa $(P>0,05)$ no tempo zero, diferenças $(P<0,05)$ nos níveis residuais de nitrito foram observadas após 12 e 27 dias de armazenamento entre mortadelas irradiadas e não-irradiadas. Menores valores de nitrito residual com a aplicação da radiação gama têm sido reportados em diversos produtos (AHN et al., 2003; HOUSER et al., 2003; AHN et al., 2004; HOUSER et al., 2005), sendo dependente da dose aplicada e do tipo de sistema de embalagem utilizado. JO et al. (2003), no entanto, não observaram redução nos valores de nitrito residual em salsichas irradiadas com $5 \mathrm{kGy}$, embaladas a vácuo ou não.

Para os teores de pigmentos heme totais (PHT) e nitrosos (PHN), foi verificado efeito significativo $(P<0,05)$ para a interação tripla entre os tratamentos (Figura 3). A redução nos teores de PHT, embora não esperada, pode ser atribuída à oxidação lipídica ocorrida nas mortadelas durante o armazenamento (Figura 1), uma vez que os radicais livres e peróxidos produzidos podem reagir com proteínas, vitaminas e pigmentos (AHN et al., 2006). Assim, em condições oxidantes, os PHN podem ser oxidados a metamioglobina, de cor marrom, com a simultânea dissociação do óxido nítrico (NO) do grupo heme (hematina).
Nas amostras adicionadas de $75 \mathrm{ppm}$ de nitrito, os teores de PHN foram menores que 30ppm após 27 dias (Figura 3A), enquanto nas amostras com $150 \mathrm{ppm}$ de nitrito estes valores somente foram alcançados após 55 dias de estocagem (Figura 3B). Esta menor redução pode ser devida à maior quantidade de nitrito residual (Figura 2A) nas amostras com maior adição deste aditivo, mantendo os teores de PHN mais elevados.

Quanto aos efeitos da irradiação sobre os pigmentos nitrosos, estes foram maiores nas amostras com 150ppm de nitrito após 27 dias de armazenamento (Figura 3B), quando os níveis de PHN foram menores $(P<0,05)$ naquelas com maiores doses de irradiação, apresentando uma redução de 14\% (para 7,5kGy) a 27\% (para 15kGy), quando comparado à amostra controle. Isso pode ser devido à maior formação de radicais livres pela radiólise da água (BREWER, 2009), que pode interagir com os PHN, contribuindo para a dissociação do NO e sua subsequente oxidação.

Também foi observado efeito $(P<0,05)$ da interação tripla entre os tratamentos para o índice de vermelho (a*). Vários trabalhos (AHN et al., 2003; JO et al., 2003; AHN et al., 2004; HOUSER et al., 2005) reportaram uma redução na cor vermelha de produtos cárneos curados irradiados, sendo atribuída à dissociação do NO do PHN e subsequente formação de metamioglobina, acarretando na descoloração (fading).

No presente trabalho, os valores de a* das amostras adicionadas de $150 \mathrm{ppm}$ de nitrito não foram afetados $(P>0,05)$ pela dose de irradiação, permanecendo constantes $\left(\mathrm{a}^{*}=16,35\right)$ durante todo $\mathrm{o}$ 


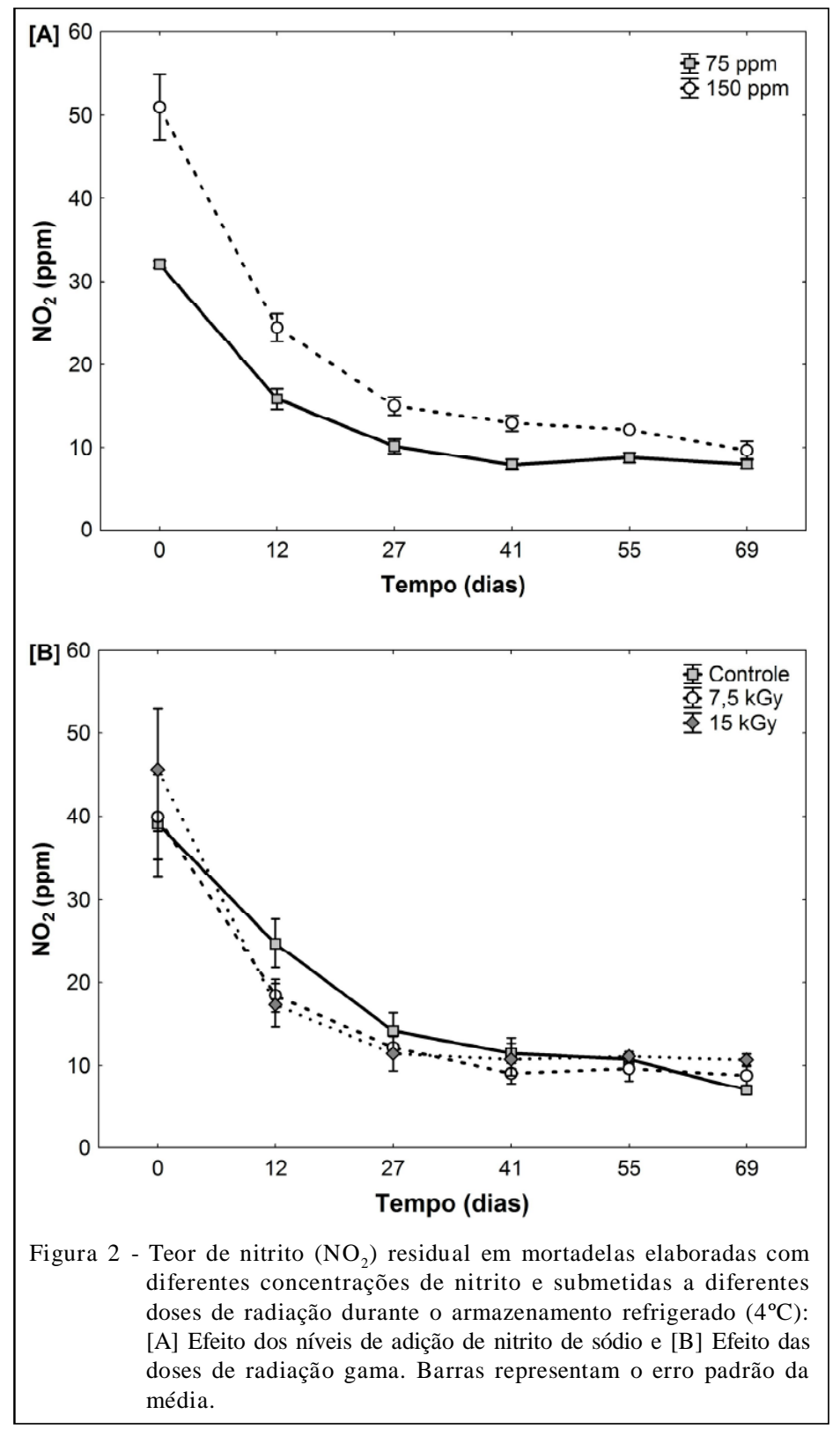

armazenamento. Nas amostras adicionadas de $75 \mathrm{ppm}$ de nitrito e irradiadas a $15 \mathrm{kGy}$, uma redução na cor vermelha pôde ser observada a partir do $27^{\circ}$ dia de estocagem (Figura 4). No entanto, tal redução não pode ser atribuída à queda dos teores de PHN (Figura $3 \mathrm{~A}$ ). Além disso, também foi observada uma queda $(P<0,05)$ nos valores de PHT a partir do 27을 dia (Figuras $3 \mathrm{~A} \mathrm{e}$ 3B), de forma que a proporção de PHN nos produtos sofreu apenas uma pequena alteração e, segundo RAMOS \& GOMIDE (2007), a cor final do produto será aquela característica da forma química predominante.

Estes resultados suportam a premissa de que a alterações na cor vermelha pela irradiação não deve ser atribuída apenas à dissociação do NO do pigmento, sendo necessários mais estudos para elucidar este fenômeno. Uma possibilidade é a considerável liberação de gás monóxido de carbono (CO) pela radiólise (AHN et al., 2006), que, devido à alta afinidade pela molécula de mioglobina (RAMOS \& GOMIDE, 2007), pode interagir com os pigmentos heme não-nitrosos, formando o pigmento de carboxihemocromo (CO-Mb), de cor vermelho brilhante, contribuindo para a manutenção dos valores de $\mathrm{a}^{*}$ do produto. Esse pigmento, no entanto, é altamente dependente das condições redutoras do meio, uma vez que o complexo $\mathrm{CO}-\mathrm{Mb}$ somente pode ser formado 

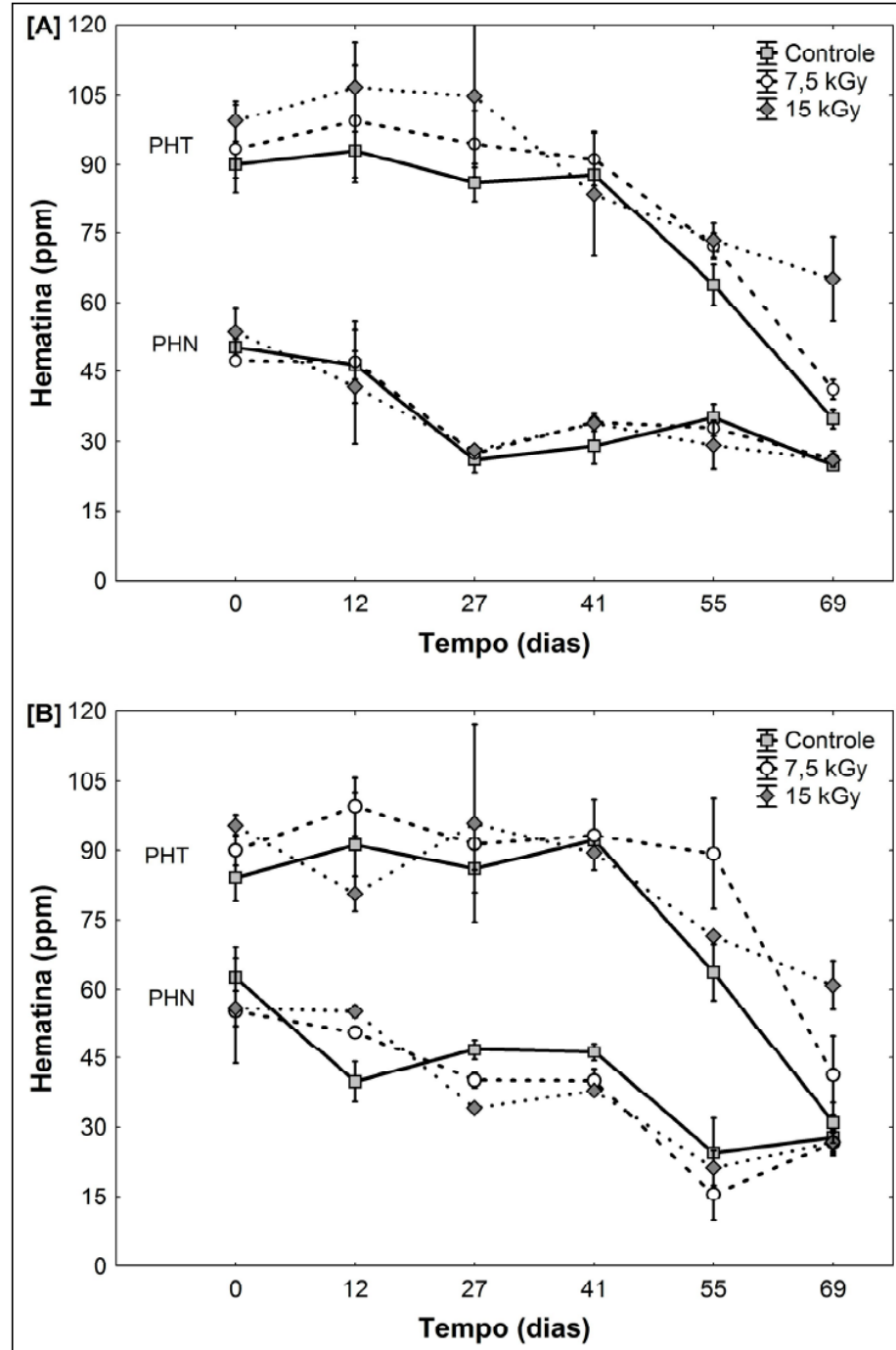

Figura 3 - Teor de pigmentos heme totais (PHT) e nitrosos (PHN) em mortadelas elaboradas com diferentes concentrações de nitrito e submetidas a diferentes doses de radiação durante $o$ armazenamento refrigerado $\left(4^{\circ} \mathrm{C}\right)$ : $[\mathrm{A}]$ Amostras adicionadas de 75ppm de nitrito de sódio e [B] Amostras adicionadas de $150 \mathrm{ppm}$ de nitrito de sódio. Barras representam o erro padrão da média.

quando o ferro heme se encontra na forma reduzida $\left(\mathrm{Fe}^{+2}\right)$ (AHN et al., 2006). Assim, com adições de 150ppm de nitrito, as condições redutoras na mortadela podem ter sido mantidas pela manutenção dos íons ferrosos $\left(\mathrm{Fe}^{+2}\right)$ e inibição da formação de íons férricos $\left(\mathrm{Fe}^{+3}\right)$, catalisadores ativos da oxidação lipídica (BREWER, 2009). Nos produtos com $75 \mathrm{ppm}$ de nitrito esta condição, no entanto, pode ter sido limitada pelos baixos valores de nitrito residual, associado a elevadas doses de irradiação (15kGy) e consequente maior radiólise, ocasionando um possível aumento no potencial de oxiredução. Segundo AHN et al. (2006), o aumento no potencial de oxi-redução facilita a conversão da forma ferrosa para a férrica, o que reduz a afinidade do CO à mioglobina e, consequentemente, diminui a intensidade da cor rosa.

A possibilidade de contribuição dos pigmentos $\mathrm{CO}-\mathrm{Mb}$ na manutenção da cor vermelha $\left(\mathrm{a}^{*}\right)$ em produtos curados é reforçada pelo comportamento deste índice nos produtos não adicionados de nitrito (Figura 4). Pode-se observar um aumento nos valores de $a^{*}$ nos primeiros dias, quando as condições redutoras ainda são altas, seguido de um decréscimo, provavelmente devido ao aumento do potencial de oxiredução com o armazenamento. HOUSER et al. (2005) observaram menores valores no potencial de oxi- 


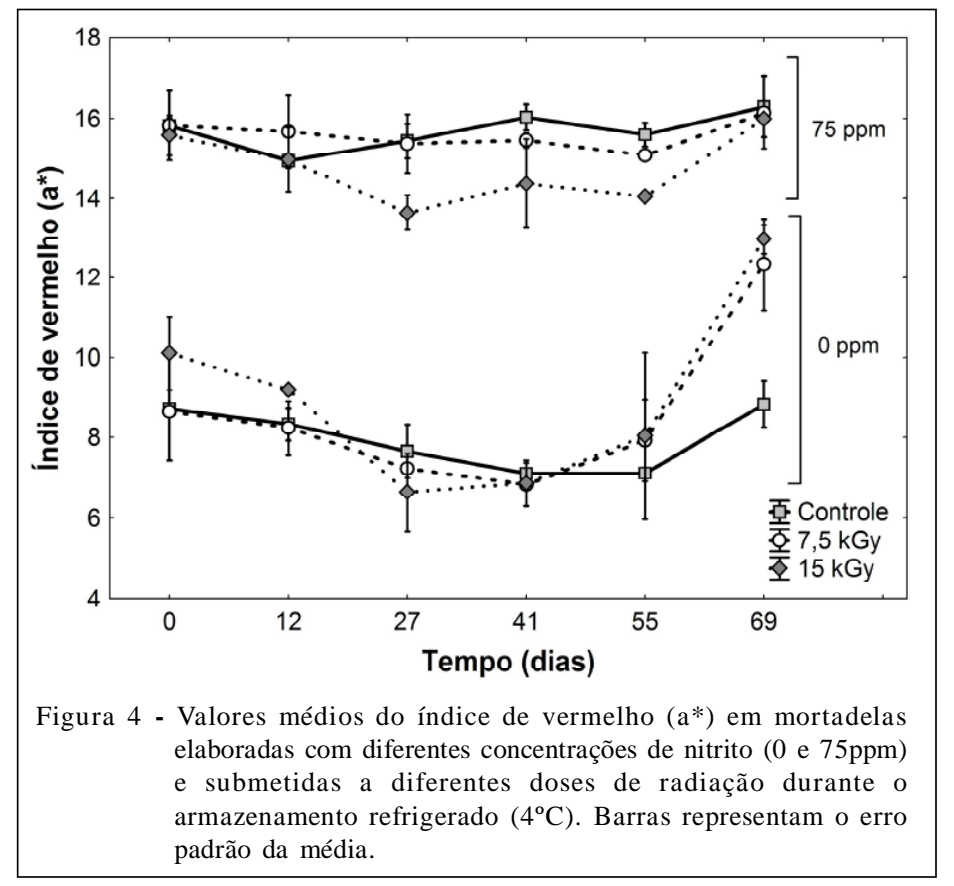

redução de presuntos irradiados (1,2 a 4,5kGy) quando comparado aos controle, sendo que estes valores aumentaram com o armazenamento $\left(4^{\circ} \mathrm{C}\right)$ por sete dias.

$\mathrm{O}$ aumento no índice de vermelho nas amostras não adicionadas de nitrito a partir do dia 55 (Figura 4) pode ser explicado pela queda dos PHT. Embora nestas amostras não tenham sido adicionado nitrito, foram detectados níveis de nitrito residual próximos de $5 \mathrm{ppm}$ no tempo zero. Este nitrito pode ter sido oriundo de uma contaminação natural do sal $(\mathrm{NaCl})$ por nitrato. Embora em pequena quantidade, a concentração de PHN nas amostras controle se elevou neste período, com comitente queda dos níveis de nitrito residual e dos valores de PHT (dados não apresentados). Assim, a relação PHN/PHT se elevou, favorecendo os valores de $\mathrm{a}^{*}$ nas amostras.

A aplicação de $15 \mathrm{kGy}$ de irradiação induziu a "regeneração" da cor vermelha das amostras adicionadas de 75ppm após 55 dias de armazenamento (Figura 4). Isso também foi observado por HOUSER et al. (2005) em presuntos cozidos, através da razão $\mathrm{a} * / \mathrm{b} *$, porém em um período de apenas sete dias de estocagem refrigerada. Estes autores não puderam atribuir o motivo desta regeneração, embora tenham concluído que não pode ser explicada pela formação de PHN, devido à conversão de nitrito residual a NO. Os resultados do presente experimento também descartam a possibilidade da formação de PHN a partir do nitrito como responsável pela regeneração da cor, mas este aumento nos valores de $\mathrm{a}^{*}$ também pode estar relacionado à queda na concentração de PHT (Figura 3). Como os
PHN sofreram apenas pequenas alterações neste período, houve uma elevação na relação PHN/PHT, indicando uma maior proporção de PHN (vermelhorosado) na formação da cor do produto.

Segundo RAMOS \& GOMIDE (2007), a perda da cor curada (fading) resulta no aumento dos valores de $a^{*}$ e/ou na redução dos valores de $b^{*} e$, portanto, pode ser acompanhada pela redução na razão $a^{*} / b^{*}$. No presente experimento, os valores de $b^{*}$ foram afetados $(P<0,05)$ apenas pela interação entre a quantidade de nitrito e o período de armazenamento, não variando com os níveis de irradiação. O índice de amarelo (b*) das amostras com 150ppm de nitrito foi constante durante todo o armazenamento $\left(b^{*}=8,23\right)$, enquanto nas amostras adicionadas de $75 \mathrm{ppm}$ de nitrito, um ligeiro aumento pôde ser observado (de 7,56 no primeiro dia a 8,18 após 69 dias de estocagem). Nas amostras não adicionadas de nitrito, houve um comportamento inverso ao observado para o índice de vermelho, sendo $b^{*}=8,38$, no primeiro dia, seguido de um aumento linear nos 40 dias seguintes até valores de 9,90 e permanecendo constantes até o último dia, quando atingiu valores médios de 9,11.

Para os valores de luminosidade $\left(\mathrm{L}^{*}\right)$, foi observado apenas efeito significativo $(P<0,05)$ dos níveis de nitrito. AHN et al. (2004) também não observaram efeito da irradiação nos valores de $\mathrm{L}^{*}$ de salsichas irradiadas (5 a 20kGy). Independentemente da irradiação, maiores valores de $\mathrm{L}^{*}(P<0,05)$ foram observados nas amostras não adicionadas de nitrito $\left(L^{*}=59,97\right)$, porém não houve diferença $(P>0,05)$ entre 
as amostras adicionadas de 75 e 150ppm deste aditivo $\left(\mathrm{L}^{*}=58,46\right)$.

BYUN et al.(2002) reportaram que a cor desejada de presuntos cozidos poderia ser obtida pela irradiação gama sem a adição de nitrito na formulação. No presente experimento, a cor desejável de mortadelas não foi possível de ser obtida sem a adição de nitrito, embora menores níveis $(75 \mathrm{ppm})$ tenham sido suficientes para manter a cor curada mesmo com a aplicação de altas doses de irradiação.

\section{CONCLUSÃO}

A aplicação de altas doses de irradiação gama promoveu a oxidação lipídica, mas não influenciou na cor curada desejável das mortadelas adicionadas de nitrito. Já a adição de 75ppm de nitrito na formulação foi suficiente para manter a cor desejada durante todo o armazenamento, independente da dose de irradiação utilizada.

\section{AGRADECIMENTOS}

Os autores agradecem à Fundação de Amparo à Pesquisa do Estado de Minas Gerais (FAPEMIG), ao apoio a Projetos Institucionais com a Participação de Recém-Doutores (PRODOC) e à Coordenação de Aperfeiçoamento de Pessoal de Nível Superior (CAPES), pelo apoio financeiro à execução do projeto.

\section{REFERÊNCIAS}

AHN, D.U. et al. Meat decontamination by irradiation. In: NOLLET, M.L.; TOLDRÁ, F. Advanced technologies for meat processing. New York: CRC Taylor e Francis, 2006. Cap.7, p.155-191.

AHN, H.J. et al. Irradiation and modified atmosphere packaging effects on residual nitrite, ascorbic acid, nitrosomyoglobin, and color in sausage. Journal of Agricultural and Food Chemistry, v.51, p.1249-1253, 2003. Disponível em: <http:/ /pubs.acs.org/doi/full/10.1021/jf025794a>. Acesso em: $07 \mathrm{fev}$. 2011.

AHN, H.J. et al. Effects of gamma irradiation on residual nitrite, residual ascorbate, color, and N-nitrosamines of cooked sausage during storage. Food Control, v.15, p.197-203, 2004. Disponível em: 〈http://dx.doi.org/10.1016/S0956-7135(03)00047-1〉. Acesso em: 07 fev. 2011.

ASSOCIATION OF OFFICIAL ANALYTICAL CHEMISTS (AOAC). Official methods of analysis of AOAC international. Virginia, 1995. V.16.
BRASIL. Ministério da Saúde. Agência Nacional de Vigilância Sanitária (ANVISA). Resolução RDC n.21, de 26 de janeiro 2001. Aprova o "Regulamento Técnico para Irradiação de Alimentos". Lex: Diário Oficial da União, 29 de janeiro de 2001. Disponível em: <http://www.anvisa.gov.br/legis/resol/ 21_01rdc.htm>. Acesso em: 13 maio, 2011.

BREWER, M.S. Irradiation effects on meat flavor: a review. Meat Science, v.81, p.1-14, 2009. Disponível em: <http:// dx.doi.org/10.1016/j.meatsci.2008.07.011>. Acesso em: 07 fev. 2011.

BREWER, M.S. Irradiation effects on meat color - a review. Meat Science, v.68, p.1-17, 2004. Disponível em: <http:// dx.doi.org/10.1016/j.meatsci.2004.02.007>. Acesso em: 07 fev. 2011

BYUN, M.W. et al. Improvement of shelf stability and processing properties of meat products by gamma irradiation. Radiation Physics and Chemistry, v.63, p.361-364, 2002. Disponível em: <http://dx.doi.org/10.1016/S0969-806X(01)00526-6>. Acesso em: 07 fev. 2011.

CASSENS, R.G. Residual nitrite in cured meat. Food Technology, v.51, p.53-55, 1997.

DONG, Q.L. et al. The effect of sodium nitrite on the textural properties of cooked sausage during cold storage. Journal of Texture Studies, v.38, p.537-554, 2007. Disponível em: <http://onlinelibrary.wiley.com/doi/10.1111/j.17454603.2007.00112.x/full>. Acesso em: 07 fev. 2011.

HOUSER, T.A. et al. Effects of irradiation on properties of cured ham. Journal of Food Science, v.68, p.2362-2365, 2003. Disponível em: <http://onlinelibrary.wiley.com/doi/ 10.1111/j.1365-2621.2003.tb05773.x/pdf>. Acesso em: 07 fev. 2011

HOUSER, T.A. et al. Irradiation-induced cured ham color fading and regeneration. Journal of Food Science, v.70, 2005. Disponível em: <http://onlinelibrary.wiley.com/doi/10.1111/ j.1365-2621.2005.tb07174.x/pdf>. Acesso em: 07 fev. 2011.

JO, C. et al. Packaging and irradiation effect on lipid oxidation, color, residual nitrite content and nitrosamine in cooked pork sausage. Food Control, v.14, p.7-12, 2003. Disponível em: <http://dx.doi.org/10.1016/S0956-7135(02)00045-2>. Acesso em: 07 fev. 2011.

PÉREZ-RODRÍGUEZ, M.L. et al. Monitoring nitrite and nitrate residues in frankfurters during processing and storage. Meat Science, v.44, p.65-73, 1996. Disponível em: <http:// dx.doi.org/10.1016/S0309-1740(96)00036-8>. Acesso em: 07 fev. 2011

RAMOS, E.M.; GOMIDE, L.A.M. Avaliação da qualidade de carnes: fundamentos e metodologias. Viçosa: UFV, 2007. 599p.

SIMIE, M.C. Radiolysis of aqueous components of food. In: JOSEPHSON, E.S.; PETERSON, H.S. Preservation of food by ionizing radiation. Boca Raton: CRC, 1983. V.II, p.8. 